\title{
The effect of quercetin on fertility of frozen-thawed ram epididymal spermatozoa
}

\author{
R. Ardeshirnia, M. Zandi ${ }^{\#}$ \& M.R. Sanjabi \\ Department of Agriculture, Iranian Research Organization for Science and Technology (IROST), P. O. Box 33535111, \\ Tehran, Iran
}

(Received 2 November 2016; Accepted 2 March 2017; First published online 13 March 2017)

\begin{abstract}
Copyright resides with the authors in terms of the Creative Commons Attribution 4.0 South African License. See: http://creativecommons.org/licenses/by/4.0/za

Condition of use: The user may copy, distribute, transmit and adapt the work, but must recognize the authors and the South African Journal of Animal Science.
\end{abstract}

\begin{abstract}
The aim of the present study was to evaluate the effects of quercetin as an antioxidant supplement on frozen-thawed ram epididymal sperm quality. Quercetin is a type of flavonoid antioxidant that is found in plants, with the ability to scavenge free radicals. Twenty testicles from mature rams were collected from a nearby slaughterhouse immediately after slaughter. Epididymal spermatozoa were recovered from the caudal of epididymides by injecting Bracket and Oliphant's (BO) medium retrogradely through the ductus deferens and extended with a tris egg-yolk-based extender and supplemented with 0, 5, 10, 15, 20, and 50 $\mu \mathrm{g} / \mathrm{mL}$ quercetin. Following equilibration, the straws were frozen, and then plunged into liquid nitrogen. After thawing, optimized concentrations of quercetin were defined based on their viabilities and used to assess fertilization and developmental potential. The results showed that the viability of frozen-thawed spermatozoa significantly increased by using 5 and $10 \mu \mathrm{g} / \mathrm{mL}$ quercetin in the freezing extender. However, total and progressive motility of frozen-thawed spermatozoa were not affected by 5 and $10 \mu \mathrm{g} / \mathrm{mL}$ quercetin in comparison with control $(0 \mu \mathrm{g} / \mathrm{mL})$. The mean number of zygote, morula, and blastocyst stage embryos increased significantly by using 5 and $10 \mu \mathrm{g} / \mathrm{mL}$ quercetin compared with other frozen-thawed treatments $(P$ $<0.05)$. However, the blastocyst rate of fresh sperm was significantly higher $(P<0.05)$. In conclusion, to improve the quality of frozen-thawed ram epididymal spermatozoa, 5 and $10 \mu \mathrm{g} / \mathrm{mL}$ quercetin appears to be an attractive option. Further studies are suggested to understand the synergistic effect of quercetin with other antioxidants to improve the ram freezing-thawing process.
\end{abstract}

Keywords: antioxidant, cryopreservation, fertility, freezing extender

\# Corresponding author: mz1075@yahoo.com

\section{Introduction}

Sperm cryopreservation is an indispensable tool to conserve the elite germplasm to improve farm animal species (Kar et al., 2015). Sperm recovery from the caudal epididymis can be advantageous after the unexpected death of endangered animals or animals with genetic merit. This procedure is an important tool for the recovery and conservation of germplasm after death (Papa et al., 2015). Additionally, there is no need to maintain male animals for the laborious process of harvesting ejaculated sperm for research, especially in sheep with seasonal reproduction. However, ejaculated sperm differ in many parameters, including the types of proteins that are bound to the plasma membrane and their motion characteristics. It is known that the in vivo fertility of epididymal sperm tends to be lower than that of ejaculated ones (Papa et al., 2015).

While cryopreservation has deleterious effects on mammalian sperm, the extent of that damage varies across species and relies heavily on sperm resilience to the freezing-thawing process (Yeste, 2016). Despite the many technical advances in the last decades, artificial insemination (AI) in sheep has not achieved the widespread use that has occurred in the cattle and swine industries. The female size, low economic return, variable fertility rates, and more rapid loss of semen quality with storage time are important obstacles for the routine use of these techniques. Therefore, sperm quality, which is negatively affected by cryopreservation, has a critical impact on the application of Al (Mata-Campuzano et al., 2014; Najafi et al., 2014).

Oxidative stress, owing to the imbalance between the presence of reactive oxygen species (ROS), such as hydrogen peroxide $\left(\mathrm{H}_{2} \mathrm{O}_{2}\right)$, and sperm antioxidant activity, is a major cause of spermatozoal damage during the freezing process. The lipid composition of the sperm plasma membrane makes this structure susceptible to oxidative damage owing to the high content in unsaturated fatty acids, especially in ram 
spermatozoa, which contain a higher polyunsaturated/saturated fatty acids ratio than other species, such as rabbits, bulls and human beings (Silva et al., 2013; Martínez-Páramo et al., 2012; Akalin et al., 2015).

Sperm protection against oxidative stress is provided mainly by seminal plasma, which contains several antioxidant components. However, the endogenous antioxidative capacity of semen is not sufficient during storage, and dilution in the extender media also reduces the seminal plasma constituents (Cabrita et al., 2011; Daramola et al., 2015). Various strategies have been used to improve the freezing-thawing process, including the usage of cryoprotectants and cryodiluents, altering the cooling and warming rates and, most importantly, supplementing semen freezing media with various antioxidants (Forouzanfar et al., 2013). Antioxidants are the main defence against oxidative stress induced by free radicals. There are preventive antioxidants and scavenger antioxidants. Preventive antioxidants, such as metal chelates and metal-binding proteins, block the formation of new free radicals, whereas scavenging antioxidants remove the free radicals that have already formed (Fathollahi et al., 2013).

In recent years, the use of herbal antioxidants has been gaining attention from researchers. Two-thirds of the world's plant species have medicinal value. In particular, many medicinal plants have great antioxidant potential (Motlagh et al., 2014). Quercetin is a flavonoid polyphenol, with more intensive antioxidant activity than vitamins $\mathrm{E}$ and $\mathrm{C}$, as well as less toxicity. Phenolic compounds act as free radical scavengers and, at times, as metal chelates, acting both in the initiation step and in propagation of the oxidative process (Silva et al., 2012).

Because of the antioxidant properties of polyphenols and the need to improve the quality of cryopreserved epididymal ram sperm, the aim of the present study was to assess the effect of various concentrations of quercetin on viability, motility and fertility of frozen-thawed ram epididymal sperm by using in vitro fertilization technique.

\section{Material and Methods}

All the culture media and other chemicals were obtained from Sigma Chemical Co. (St. Louis, Mo, USA) and the plasticwares were purchased from Falcon (Paignton, UK), unless stated otherwise.

Testes with epididymides from 10 chal rams (2.5-3.5 years of age) were collected from a nearby slaughterhouse immediately after slaughter. In the laboratory, the testes were rinsed twice with normal saline and were then trimmed to remove the extra testicular tissue and washed 10 times with saline containing $0.1 \%$ streptomycin sulphate. Connective tissue covering the caudal epididymis was removed by dissection with care to avoid rupturing blood vessels or the epididymal duct. Spermatozoa were recovered from the caudal of epididymides by injecting BO medium (containing $10 \mu \mathrm{g} / \mathrm{mL}$ heparin, $137.0 \mu \mathrm{g} / \mathrm{mL}$ sodium pyruvate, $1.942 \mathrm{mg} / \mathrm{mL}$ caffeine sodium benzoate, and $10 \mathrm{mg} / \mathrm{mL}$ fatty acid free bovine serum albumin (BSA)) retrogradely through the ductus deferens. Collected spermatozoa were washed once by centrifugation with BO medium at $600 \mathrm{~g}$ for $7 \mathrm{~min}$. The pellet obtained was suspended in $\mathrm{BO}$ medium and evaluated for motility.

The extender in the present study was prepared according to the protocol established by Baghshahi et al. (2014). The extender was composed of $2.7 \mathrm{~g}$ tris, $2.44 \mathrm{~g}$ citric acid, $0.5 \mathrm{~g}$ glucose, $7 \%$ glycerol (v/v), $15 \%$ egg yolk and $100 \mathrm{IU}$ penicillin for $100 \mathrm{~mL}$ sterile Milli-Q water. The osmolarity and pH were set at $335 \mathrm{mOsm}$ and 6.5, respectively. The final treatments were prepared using various concentrations of quercetin (0-50 $\mu \mathrm{g} / \mathrm{mL}$ ) added to prepared extenders. The spermatozoa samples from six testes (one testis from each ram) with more than $80 \%$ motility were pooled and diluted with different extenders prepared at $25^{\circ} \mathrm{C}$ to a final concentration of $4 \times 10^{8} \mathrm{sperm} / \mathrm{mL}$. The samples were drawn into $0.25 \mathrm{~mL}$ French straws (IMV, L'Aigle, France) which were maintained at $4{ }^{\circ} \mathrm{C}$ for two hours. The straws were then placed $3 \mathrm{~cm}$ above the surface of liquid nitrogen, where the temperature was approximately $-120{ }^{\circ} \mathrm{C}$. After 15 minutes, the straws were immersed directly into liquid nitrogen $\left(-196{ }^{\circ} \mathrm{C}\right)$ for long-time storage. After one month, three straws were thawed in a $37^{\circ} \mathrm{C}$ water bath for 30 seconds, pooled, and immediately subjected to analysis and evaluation.

The MTT assay was performed according to the protocol established by Byun et al. (2008) with some modification. This assay was used to access sperm viability. For each sample, six wells of the 96-well microplate were used. One hundred $\mathrm{mL}$ sperm sample $\left(30 \times 10^{6} \mathrm{sperm} / \mathrm{mL}\right)$ plus $10 \mu \mathrm{L}$ of MTT (Thermo Fisher Scientific, USA,V-13154) stock solution [5 mg MTT/mL Phosphate-buffered saline (PBS)] were placed in each well. Spermatozoa were incubated at $37^{\circ} \mathrm{C}$ in a humidified atmosphere for four hours. Then $100 \mu \mathrm{L}$ of the sodium dodecyl sulphate (SDS) - hydrogen chloride $(\mathrm{HCl})$ solution $(10 \mathrm{~mL}$ of $0.01 \mathrm{M} \mathrm{HCl}$ was added to $1 \mathrm{~g} \mathrm{SDS}$ ) was added to each well and mixed thoroughly using the pipette and spermatozoa were incubated at $37^{\circ} \mathrm{C}$ for 16 hours in a humidified chamber. The absorbance was measured in a spectrophotometer (MS2 Reader). The freeze-killed procedure was used to obtain the standard curve and the relationship between the MTT reduction rate and sperm viability. After the dilution of sperm with BO medium, $3 \mathrm{~mL}$ of the diluted sperm was divided in two fractions. One fraction was maintained at $37^{\circ} \mathrm{C}$, while the sperm in the other fraction were killed by two cycles of plunging into liquid nitrogen and thawing at $37^{\circ} \mathrm{C}$. Treatments for 
analysis were prepared by combining aliquots of viable and freeze-killed spermatozoa at ratios of 10:0, 8:2, $6: 4,4: 6,2: 8$, and $0: 10 \mathrm{v} / \mathrm{v}$, respectively. The prepared samples were analysed by the MTT assay (above). Standard curves, $y=0.0139 x-1.0108$ (where $y$ and $x$ stand for optical density and viability, respectively) for the relationship between the MTT reduction rate and the percentage of viable spermatozoa were acquired (Figure 1). This standard curve was applied to acquire the percentage of viable sperm in each sample in accordance with the MTT reduction rate.

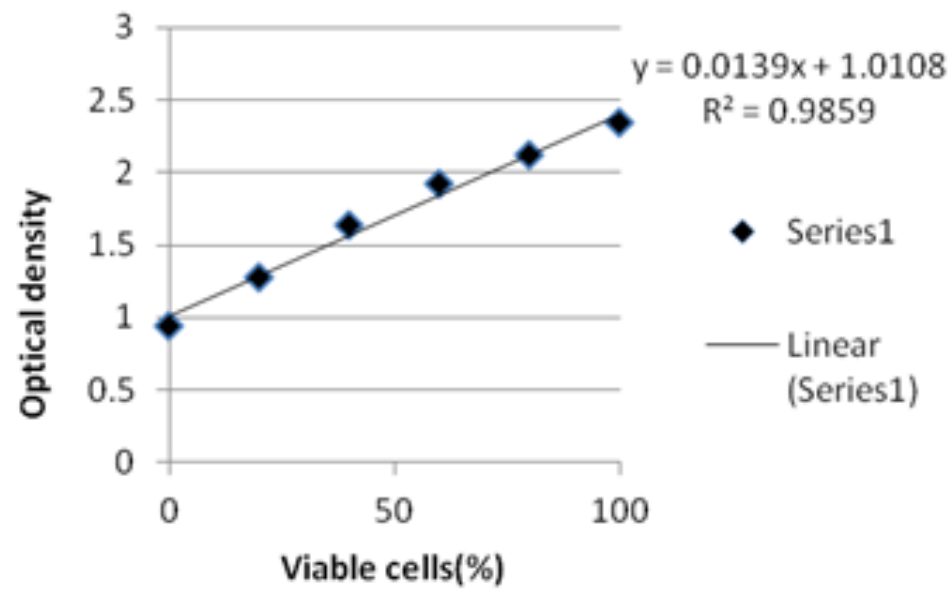

Figure 1 Relation between viability of spermatozoa and their optical density by using MTT assay

Ten $\mathrm{mL}$ sperm were placed on a slide $\left(37^{\circ} \mathrm{C}\right)$. Spermatozoa with motility and progressive motility were then assessed by counting 200 spermatozoa in each replicate using bright-field microscopy (400x) (Olympus CX21FS1, Japan). Each sample was counted 10 times. Based on the World Health Organization's (WHO) laboratory manual (2010) the spermatozoa moving actively, either linearly or in a large circle, regardless of speed, were graded as having progressive motility.

In vitro embryo production was performed according to the protocol established by Zandi et al. (2014) with some modification. Sheep ovaries collected from a nearby abattoir were brought over to the laboratory in PBS containing $100 \mathrm{IU} / \mathrm{mL}$ penicillin and $50 \mathrm{mg} / \mathrm{mL}$ streptomycin at 30 to $34{ }^{\circ} \mathrm{C}$ within three hours of slaughter. Cumulus-oocyte complexes (COCs) were released from all visible follicles (>2 $\mathrm{mm}$ diameter) by slicing the surface of an ovary with a surgical blade. Those with more than three layers of compact unexpanded cumulus cells and homogenous evenly granular ooplasm were transferred to $100 \mathrm{~mL}$ droplets (15 to 20 COCs per droplet) of in vitro maturation (IVM) medium, which consisted of TCM-199, containing $10 \%$ foetal bovine serum (FBS), $10 \%$ buffalo follicular fluid, $5 \mathrm{mg} / \mathrm{mL}$ follicle stimulating hormone (FSH), 1 $\mathrm{mg} / \mathrm{mL}$ oestradiol-17ß, $0.81 \mathrm{mM}$ sodium pyruvate, and $50 \mathrm{mg} / \mathrm{mL}$ gentamicin sulphate, and were cultured under mineral oil in a petri dish in a $\mathrm{CO}_{2}$ incubator at $38.5^{\circ} \mathrm{C}$ for 24 hours. For in vitro fertilization the oocytes were washed twice with $\mathrm{BO}$ medium and were transferred to $50 \mathrm{~mL}$ droplets (15 to 20 oocytes per droplet) of capacitation and fertilization BO medium [washing BO medium containing $10 \mathrm{mg} / \mathrm{mL}$ fatty acid-free bovine serum albumin (BSA)]. The spermatozoa were prepared for fertilization according to the protocol established by Chauhan et al. (1998). Oocytes were then inseminated with a final concentration of $1 \times 10^{6} / \mathrm{mL}$ motile spermatozoa and incubated under mineral oil in a $\mathrm{CO}_{2}$ incubator at $38.5^{\circ} \mathrm{C}$ for 18 hours. The cumulus cells were then removed from the presumed zygotes, after which these were cultured in mCR2aa containing $0.6 \%$ BSA for 48 hours in groups of 15 to20 per droplet. Following this, the cleaved embryos obtained were cultured in the in vitro culture (IVC) medium (mCR2aa+0.6\% BSA+10\% FBS) for up to 8 days until morulae and blastocysts were obtained, with a change of medium every 48 hours. To study the fertility of fresh and frozen-thawed spermatozoa each treatment was replicated six times.

Data were analysed with a statistical software program (SPSS 16, IBM, USA). Comparisons between multiple numeric datasets were performed using one-way ANOVA followed by Duncan's multiple-range test. Results are expressed as mean \pm SEM, and statistical significance was accepted at $P<0.05$.

The statistical model used was:

$Y_{i j}=\mu+a_{i}+e_{i j}$ 
Where: $Y_{i j}$ is the trait under study or response variables (viability of fresh spermatozoa or viability, motility or fertility of frozen-thawed spermatozoa)

$\mu$ is the overall mean

$a_{i}$ is the fixed effect of different quercetin concentrations $(i=0,5,10,15,20,50$ and 100 $\mu \mathrm{g} / \mathrm{mL}$ for fresh spermatozoa, ori $=0,5,10,15,20$ and $50 \mu \mathrm{g} / \mathrm{mL}$ for frozen-thawed spermatozoa)

$\mathrm{e}_{i j}$ is the random error effect.

\section{Results}

In the first experiment, the effects of various concentrations of quercetin (0-100 $\mu \mathrm{g} / \mathrm{mL})$ on viability of fresh spermatozoa were studied. Results showed that by increasing the concentrations of quercetin from 20 $\mu \mathrm{g} / \mathrm{mL}$, the viability of spermatozoa decreased significantly $(P<0.05)$ (Figure 2). However, after the cryopreservation of sperm, 5 and $10 \mu \mathrm{g} / \mathrm{mL}$ of quercetin significantly increased the viability of spermatozoa in compared to 0,20 and $50 \mu \mathrm{g} / \mathrm{mL}$ treatments $(P<0.05)$ (Figure 3 ).

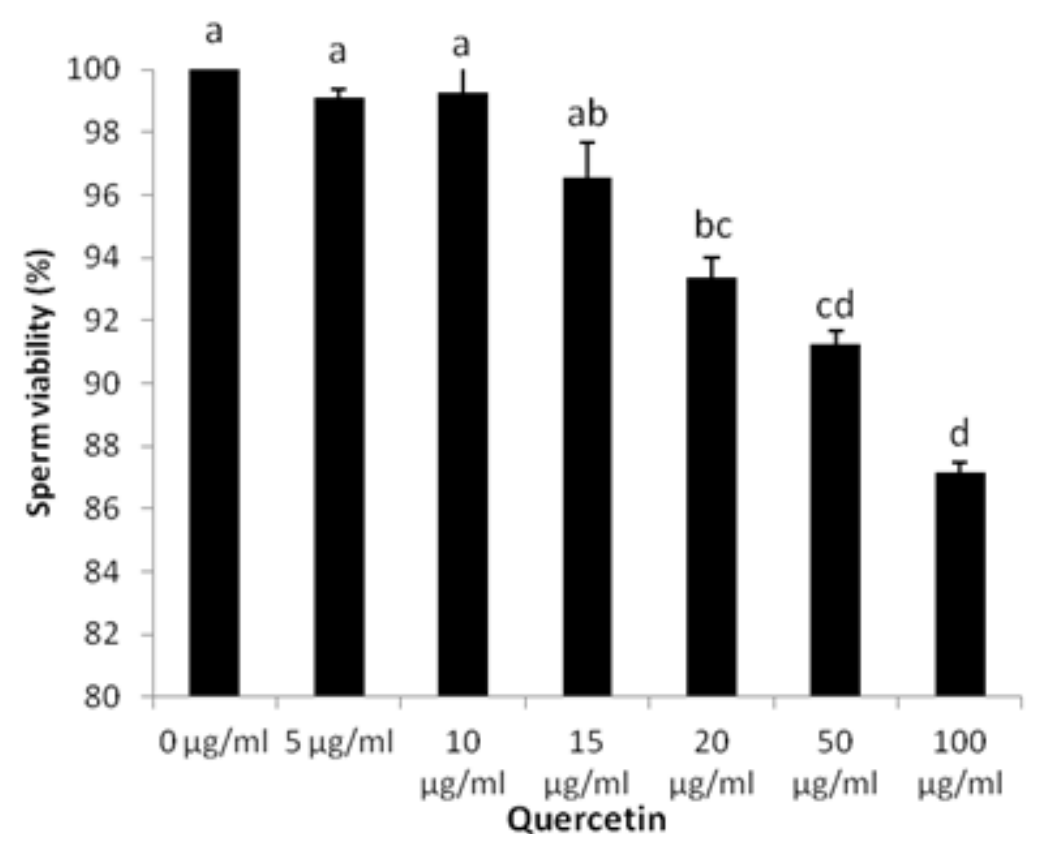

Figure 2 Effects of various concentrations of quercetin on viability of fresh spermatozoa

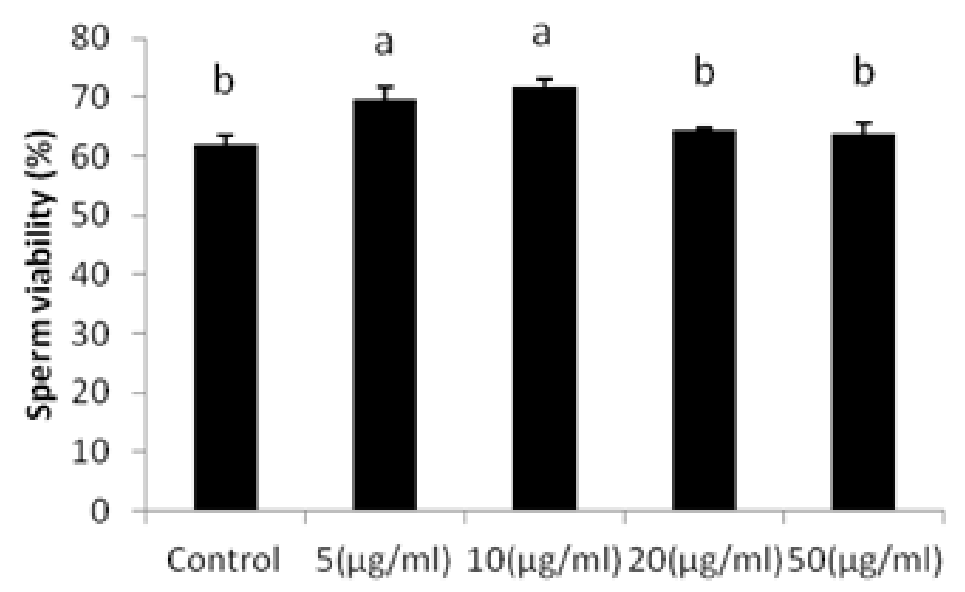

Treatments

Figure 3 Effects of various concentrations of quercetin on viability of spermatozoa after freezing and thawing 
Total motility and progressive motility of frozen-thawed spermatozoa with the addition of 5 and 10 $\mu \mathrm{g} / \mathrm{mL}$ of quercetin were not affected in comparison with $0 \mu \mathrm{g} / \mathrm{mL}$, although they decreased significantly with 20 and $50 \mu \mathrm{g} / \mathrm{mL}$ of quercetin (Figure 4).

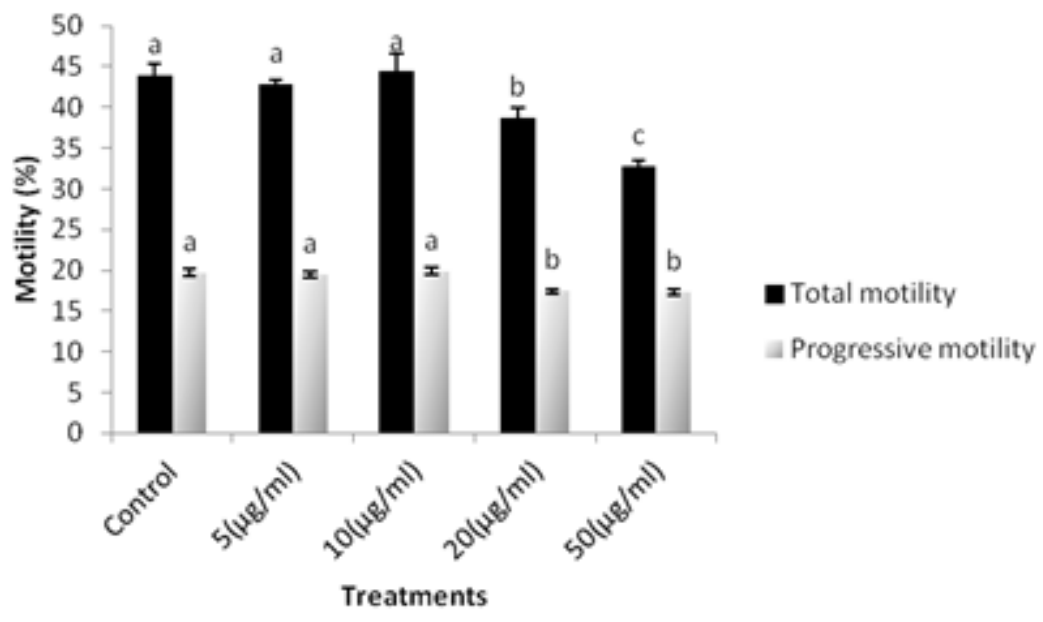

Figure 4 Effects of various concentrations of quercetin on total motility and progressive motility of spermatozoa after freezing and thawing

The fertility of frozen-thawed spermatozoa was evaluated by in vitro fertilization technique (Table 1). The mean number of zygote, morula and blastocyst stage embryos increased significantly with using 5 and $10 \mu \mathrm{g} / \mathrm{mL}$ of quercetin in comparison with other frozen-thawed treatments $(P<0.05)$. However, the blastocyst rate of fresh sperm was significantly higher than for other groups $(P<0.05)$.

Table1 Effects of quercetin in sperm extender on cleavage, morula and blastocyst development of sheep oocytes cultured in vitro

\begin{tabular}{lccc}
\hline Treatments & Cleavage (\%) & Morula(\%) & Blastocyst (\%) \\
\hline Fresh sperm & $83^{\mathrm{a}} \pm 1$ & $74^{\mathrm{a}} \pm 2$ & $37^{\mathrm{a}} \pm 1$ \\
$\mathrm{CS}^{*}(0 \mu \mathrm{g} / \mathrm{mL}$ quercetin) & $62^{\mathrm{c}} \pm 3$ & $49^{\mathrm{c}} \pm 6$ & $21^{\mathrm{c}} \pm 2$ \\
CS $(5 \mu \mathrm{g} / \mathrm{mL}$ quercetin) & $76^{\mathrm{ab}} \pm 0.5$ & $66^{\mathrm{ab}} \pm 0.3$ & $32^{\mathrm{b}} \pm 0.1$ \\
CS $(10 \mu \mathrm{g} / \mathrm{mL}$ quercetin) & $73^{\mathrm{b}} \pm 2$ & $64^{\mathrm{b}} \pm 2$ & $29^{\mathrm{b}} \pm 1$ \\
CS $(20 \mu \mathrm{g} / \mathrm{mL}$ quercetin) & $60^{\mathrm{c}} \pm 2$ & $51^{\mathrm{c}} \pm 2$ & $20^{\mathrm{c}} \pm 3$ \\
CS $(50 \mu \mathrm{g} / \mathrm{mL}$ quercetin) & $60^{\mathrm{c}} \pm 1$ & $46^{\mathrm{c}} \pm 1$ & $11^{\mathrm{d}} \pm 1$
\end{tabular}

CS: Cryopreserved sperm

Data were expressed as mean \pm SEM

Around 130 oocytes were used in each treatment

${ }_{\mathrm{a}, \mathrm{b}, \mathrm{c}}$ Different letters indicate statistical difference within each column $(P<0.05)$

\section{Discussion}

In vitro embryo production can be a valuable technique in reproductive biotechnology in farm animals. However, the feasibility and the quality of the produced ovine embryos are lower than for other species (Romao et al., 2013).This is mainly because ram spermatozoa are sensitive to the conditions of lowtemperature anabiosis and freezing (Sabev et al., 2006). Although semen possesses an antioxidant system, cryopreservation increases the intensity of lipid peroxidation of spermatozoa and their activity is affected (Najafi et al., 2014). Previous results showed that supplementation with antioxidants such as cysteamine, 
ergothioneine, vitamin $\mathrm{E}$ analogue, Trolox, catalase, and superoxide dismutase mimetic agent reduces lipid peroxidation and $\mathrm{H}_{2} \mathrm{O}_{2}$ generation (Forouzanfar et al., 2013). Increasing interest is currently being shown in the antioxidant activity of natural antioxidants and synthetic substances, such as clove bud, rosemary, resveratrol, and quercetin (Silva et al., 2012; Baghshahi et al., 2014; Motlagh et al., 2014). Research has shown that the antioxidant activity of polyphenols is more effective and less toxic than vitamins $E$ and $C$. However, various factors, such as method of administration, dose, and structure of phenolic compounds, affect their activities (Silva et al., 2012). For example, antioxidants might have negative effects owing to excessive scavenging of free radicals, possibly by altering their physiological levels (Najafi et al., 2014). As shown in the current study, viability, motility and fertility of spermatozoa decreased significantly when higher concentrations of polyphenol quercetin $(\geq 20 \mu \mathrm{g} / \mathrm{mL})$ were used.

The viability of frozen-thawed spermatozoa increased significantly by using 5 and $10 \mu \mathrm{g} / \mathrm{mL}$ quercetin in the freezing extender. However, total motility and progressive motility of frozen-thawed spermatozoa with 5 and $10 \mu \mathrm{g} / \mathrm{mL}$ of quercetin were not affected in comparison with $0 \mu \mathrm{g} / \mathrm{mL}(42 \pm 19 \%$ and $44 \pm 19 \%$ vs. $43 \pm 19 \%)$. In agreement to the current results, in a study on red deer epididymal spermatozoa after freezing and thawing. Anel-López et al. (2012) showed that despite obtaining good total motility after thawing $(60 \pm 3 \%)$, the progressive motility of the samples was very low, with only $4 \pm 0.3 \%$ of the spermatozoa being identified as progressive. In the study of Silva et al. (2012), the progressive motility on ram ejaculated spermatozoa was $50 \pm 3 \%$. These authors showed that there were no differences among experimental groups in progressive motility when using $5,10,15$ and $20 \mu \mathrm{g} / \mathrm{mL}$ of quercetin. They also reported that there were no differences among experimental groups with regard to vigour, acrosome integrity and plasma membrane integrity. The percentage of sperm with a high mitochondrial membrane potential was greater in $0 \mu \mathrm{g} / \mathrm{mL}$, then $5 \mu \mathrm{g} / \mathrm{mL}$ quercetin groups than those of 10,15 , and $20 \mu \mathrm{g} / \mathrm{mL}(P<0.05)$. That mitochondrial membrane potential values decreased with increasing concentrations of resveratrol or quercetin was probably beneficial to sperm. The mitochondrial system is the main source of intracellular ROS, with the respiratory chain acting in an autoxidation process, whereas polyphenols, such as quercetin, have important roles in absorption and neutralization of ROS, because of their oxido reduction properties (Silva et al., 2012). Bucak et al. (2007) showed that adding $5 \mathrm{mM}$ of cysteamine to a ram freezing extender yielded a higher percentage of viable spermatozoa after thawing, although they did not find any improvement in plasma membrane functional integrity. However, Çoyan et al. (2011) found that $4 \mathrm{mM}$ ergothioneine, despite the good motility results, reduced membrane integrity and mitochondrial activity.

Commonly, fertilization rate is considered the gold standard for assessing the cryopotential of semen cryopreservation media (Forouzanfar et al., 2013). The current results showed that the freezing-thawing process of spermatozoa negatively affected the mean number of cleavage, morula, and blastocyst. However, the mean number of zygote, morula and blastocyst stage embryos increased significantly when using 5 and $10 \mu \mathrm{g} / \mathrm{mL}$ of quercetin compared with other frozen-thawed treatments $(P<0.05)$. Peris et al. (2004) suggest that the poorer fertilizing efficiency of frozen ram semen might be at least partly due to the abnormal sperm DNA structure, despite having a normal appearance soon after thawing. Additionally, if the antioxidant capacity of the extender is insufficient, excessive ROS could attack sperm membranes, which are very susceptible to oxidants (Najafi et al., 2014). Quercetin inhibits ROS formation by enzymatic and nonenzymatic systems, especially NADPH oxidase and $\mathrm{NADH}$-dependent oxidoreductase, localized in the sperm plasma membrane and mitochondria, and scavenge ROS. This antioxidant induces calcium release into the cell, according to the time and concentrations of polyphenol, with control of intracellular free calcium concentration being crucial for the maintenance of normal cell function (Silva et al., 2012).

\section{Conclusions}

To improve the quality of frozen-thawed ram epididymal spermatozoa, 5 and $10 \mu \mathrm{g} / \mathrm{mL}$ quercetin appears as an attractive option. However, the blastocyst rate of fresh sperm was significantly higher. Further studies to understand the synergistic effect of quercetin with other antioxidants in order to improve the ram freezing-thawing process are suggested.

\section{Acknowledgments}

We would like to thank Dr Majed Masoumian and Amir Hossin Ahadi for their helpful comments during the research. This project supported by Iran National Science Foundation (Grant No. 93015593).

\section{Authors' Contributions}

RA was involved in the experimental design, data collection and data analysis. MZ was involved in study design, data analysis, data interpretation and manuscript editing. MRS was involved in study design and data interpretation. All authors have read and approved the final manuscript. 


\section{Conflict of Interest Declaration}

Authors declare that there is no conflict of interest for this study.

\section{References}

Akalin, P.P., Bülbül, B., Coyan, K., Baspinar, N., Bucak, M.N., Güngör, S. \& Öztürk, C., 2015. Relationship of blood and seminal plasma ceruloplasmin, copper, iron and cadmium concentrations with sperm quality in Merino rams. Small. Rumin. Res. 133, 135-139.

Anel-Lopez, L., Álvarez-Rodríguez, M., García-Álvarez, O., Álvarez, M., Maroto-Morales, A., Anel, L., de Paz, P., Garde, J.J. \& Martínez-Pastor, F., 2012. Reduced glutathione and Trolox (vitamin E) as extender supplements in cryopreservation of red deer epididymal spermatozoa. Anim. Reprod. Sci. 135, 37-46.

Baghshahi, H., Riasi, A., Mahdavi, A.H. \& Shirazi, A., 2014. Antioxidant effects of clove bud (Syzygiumaromaticum) extract used with different extenders on ram spermatozoa during cryopreservation. Cryobiology 69, 482-487.

Bucak, M.N., Atessahin, A., Varisli, O., Yuce, A., Tekin, N. \& Akcay, A., 2007. The influence of trehalose, taurine, cysteamine and hyaluronan on ram semen: Microscopic and oxidative stress parameters after freeze-thawing process. Theriogenology 67, 1060-1067.

Byun, J.W.,Choo, S.H., Kim, H.H., Kim, Y.J., Hwang, Y.J. \& Kim, D.K., 2008.Evaluation of boar sperm viability by MTT reduction assay in Beltsville thawing solution extender. Asian-Aust. J. Anim. Sci. 21, 494-498.

Cabrita, E., Ma, S., Diogo, P., Martínez-Páramo, S., Sarasquet, C. \& Dinis, M.T., 2011. The influence of certain aminoacids and vitamins on post-thaw fish sperm motility, viability and DNA fragmentation. Anim. Reprod. Sci. 125, 189-195.

Chauhan, M.S., Singla, S.K., Palta, P., Manik, R.S. \& Madan, M.L., 1998. In vitro maturation and fertilization and subsequent development of buffalo (Bubalusbubalis) embryo: Effect of oocyte quality and type of serum. Reprod. Fertil. Dev. 10, 173-177.

Çoyan, K., Bas_pinar, N., Bucak, M.N. \& Akalin, P.P., 2011. Effects of cysteine and ergothioneine on post-thawed Merino ram sperm and biochemical parameters. Cryobiology 63, 1-6.

Daramola, J.O., Adekunle, E.O., Oke, O.E.,Onagbesan, O.M., Lyasere, O.S., Williams, T.J., James, I.J., Oyewusi, I.K. \& Oyewusi, J.A., 2015. Effects of pyridoxine supplementation or in combination with other antioxidants on motility, in vitro capacitation and acrosome reaction of goat buck spermatozoa during cryopreservation. Small Rumin. Res. 131, 113-117.

Fathollahi, A., Jasemi, M. \& Saki, G., 2013. Effect of noise stress on male rat fertility, and the protective effect of vitamins $\mathrm{C}$ and $\mathrm{E}$ on its potential effect. Arab J. Urology. 11, 101-105.

Forouzanfar, M., Abid, A., Hosseini, S.M., Hajian, M. \& Nasr-Esfahani, M.H., 2013. Supplementation of sperm cryopreservation media with cell permeable superoxide dismutase mimetic agent (MnTE) improves goat blastocyst formation. Cryobiology 67, 394-397.

Kar, S., Divyashree, B.C. \& Roy, SC., 2015. Temporal leakage of Cu,Zn superoxide dismutase and loss of two lowmolecular-weight forms of glutathione peroxidase-1 from buffalo (Bubalusbubalis) sperm after freezing and thawing. Theriogenology 83, 512-519.

Martínez-Páramo, S., Diogo, P., Dinis, M.T., Herráez, M.P., Sarasquete, C. \& Cabrita, E., 2012. Incorporation of ascorbic acid and $\alpha$-tocopherol to the extender media to enhance antioxidant system of cryopreserved sea bass sperm. Theriogenology 77, 1129-1136.

Mata-Campuzano, M., Álvarez-Rodríguez, M., Tamayo-Canul, J., López-Urueña, E., de Paz, P., Anel, L., MartínezPastor, F. \& Álvarez, M., 2014. Refrigerated storage of ram sperm in presence of Trolox and GSHantioxidants: Effect of temperature, extender and storagetime. Anim. Reprod. Sci. 151, 137-147.

Motlagh, M.K., Sharafi, M., Zhandi, M., Mohammadi-Sangcheshmeh, A., Shakeri, M.,Soleimani, M. \& Zeinoaldini, S. 2014. Antioxidant effect of rosemary (Rosmarinusofficinalis L.) extract in soybean lecithin-based semen extender following freeze-thawing process of ram sperm. Cryobiology 69, 217-222.

Najafi, A., Kia, H.D., Mohammadi, H., Najafi, M.H., Sharafi, M., Martinez-Pastor, F., Adeldust, H., Zanganeh, Z., Sharafi, M., Martinez-Pastor, F. \& Adeldust, H., 2014. Different concentrations of cysteamine and ergothioneine improve microscopic and oxidative parameters in ram semen frozen with a soybean lecithin extender. Cryobiology 69, 6873.

Papa, P.M., Papa, F.O., Oliveira, L.A., Guasti, P.N., Castilho, C. \& Giometti, I.C., 2015.Different extenders in the cryopreservation of bovine epididymal spermatozoa. Anim. Reprod. Sci. 161, 58-63.

Peris, S.I., Morrier, A., Dufour, M. \& Bailey, J.L., 2004. Cryopreservation of ram semen facilitates sperm DNA damage: Relationship between sperm andrological parameters and the sperm chromatin structure assay. J. Androl. 25, 224-233.

Romao, R., Marques, C.C., Baptista, M.C., Vasques, M.I., Barbas, J.P., Carolino, N., Bettencourt, E., Plancha, C., Rodrigues, P., Pereira, R.M. \& Horta, A.E.M., 2013. Evaluation of two methods of in vitro production of ovine embryos using fresh or cryopreserved semen. Small Rumin. Res. 110, 36-41.

Sabev, M., Nikolov, I., Ivanova-kicheva, M., Stefanov, R., Chemshirova, T., Baycheva, E. \& Popova, M. 2006. Cryopreservation of ram sperm from autochthonous breeds during a non-mating season. J. Central European Agriculture 4, 677-682.

Silva, E.C.B., Cajueiro, J.F.P., Silva, S.V., Soares, P.C. \& Guerra, M.M.P., 2012. Effect of antioxidants resveratrol and quercetin on in vitro evaluation of frozen ram sperm. Theriogenology $77,1722-1726$.

Silva, S.V., Soares, A.T., Batista, A.M., Almeida, F.C., Nunes, J.F., Peixoto, C.A. \& Guerra, M.M.P., 2013. Vitamin E (Trolox) addition to Tris-egg yolk extender preserves ram spermatozoa on structure and kinematics after cryopreservation. Anim. Reprod. Sci. 137, 37-44. 
WHO laboratory manual for the examination and processing of human semen, 2010. 5th edition. ISBN 978924154778 9.

Yeste, M., 2016. Sperm cryopreservation update: Cryodamage, markers and factors affecting the sperm freezability in pigs. Theriogenology 85, 47-64.

Zandi, M., Muzaffar, M., Shah, S.M., Kaushik, R., Singh, M.K., Palta, P., Singla, S.K., Manik, R.S. \& Chauhan, M.S., 2014.WNT3A signalling pathway in buffalo (Bubalus bubalis) embryonic stem cells. Reprod. Fertil. Dev. 26, 55161. 